\title{
New Statistical Models for Randoms-Precorrected PET Scans
}

\author{
Mehmet Yavuz and Jeffrey A. Fessler \\ Department of EECS, University of Michigan, Ann Arbor MI
}

\begin{abstract}
PET measurements are usually precorrected for accidental coincidence events by real-time subtraction of the delayed window coincidences. Randoms subtraction compensates in mean for accidental coincidences but destroys the Poisson statistics. We propose and analyze two new approximations to the exact log-likelihood of the precorrected measurements, one based on a "shifted Poisson" model, the other based on saddle-point approximations to the measurement probability mass function (pmf). The methods apply to both emission and transmission tomography; however in this paper we focus on transmission tomography. We compare the new models to conventional data-weighted least squares (WLS) and conventional maximum likelihood (based on the ordinary Poisson (OP) model) using simulations and analytic approximations. The results demonstrate that the proposed methods avoid the systematic bias of the WLS method, and lead to significantly lower variance than the conventional OP method. The saddle-point method provides a more accurate approximation to the exact log-likelihood than the WLS, OP and shifted Poisson alternatives. However, the simpler shifted Poisson method yielded comparable bias-variance performance in the simulations. The new methods offer improved image reconstruction in PET through more realistic statistical modeling, yet with negligible increase in computation over the conventional OP method.
\end{abstract}

\section{Introduction}

In PET measurements, accidental coincidence $(\mathrm{AC})$ events are a primary source of background noise. AC events occur when photons that arise from separate annihilations are mistakenly registered as having arisen from the same annihilation. In transmission scans the photons that originate from different transmission sources (rod or sector sources rotating around the patient) cause $\mathrm{AC}$ events. The ratio of total $\mathrm{AC}$ events to "true" events is usually small in transmission scans compared to emission scans. Nevertheless, the effect of AC events becomes severe for regions of high attenuation coefficients, because projections through such regions result in low true coincidence rates. These low count rates can become comparable to $\mathrm{AC}$ rates. Thus estimates of the $\mathrm{AC}$ events are needed. One can use the "singles" method [1] for this purpose, however this approach is not

This work was supported in part by NIH grants CA-60711 and CA-54362. 
widely used because of the necessity for additional hardware and moreover usually singles rate vary during data acquisition [9]. Therefore, in most PET scans, the $\mathrm{AC}$ rates are estimated using delayed-window coincidences and the data are precorrected for $\mathrm{AC}$ events by real-time subtraction. Real-time subtraction of delayed window coincidences compensates in mean for $\mathrm{AC}$ events but destroys the Poisson statistics [7]. To avoid this problem, one needs to maintain the transmission and randoms measurements as two separate sinograms $[8,10]$. However even if a PET system allows one to collect randoms (delayed coincidences) sinogram separately, this process would double the storage space for the acquired data. So in practice most PET centers collect and archive only the randoms precorrected data. We recommend separate acquisition and storage of delayed coincidences wherever feasible. The purpose of this paper is to provide accurate statistical methods for PET measurements with pre-subtracted delayed coincidences. Although our analysis and proposed models apply to both emission and transmission tomography, in this paper we focus on transmission tomography.

The exact log-likelihood for randoms precorrected data is intractable, so we describe and compare several approximations. For completeness, we first review the data-weighted least squares (WLS) method and the log-likelihood for the ordinary Poisson (OP) model for PET measurements. Then, we introduce a new "shifted" Poisson (SP) model [14] which matches both the first and second-order moments of the model to the underlying statistics of the precorrected data. We derive approximate analytic expressions for the variance of the different estimators and use the Cauchy-Schwarz inequality to show analytically that the proposed SP method yields lower variance than the OP method.

Secondly, we introduce a new saddle-point (SD) approximation for the pmf of precorrected measurements. The corresponding log-likelihood function is shown to have better agreement with the exact log-likelihood than the previous approximations. We apply the fast grouped-coordinate ascent algorithm [3] (with a few simple modifications) to maximize the proposed saddle-point objective function.

We also show results of 2D simulations showing that the WLS method leads to systematic bias and the OP method leads to higher variance than SP and SD methods. We also observe that SP and SD methods yield equivalent bias/variance performance whereas SP requires less computation. The contribution of this work lies in the fact that the proposed methods offer significant improvements in accuracy with minor computation increase.

\section{Measurement Model}

In conventional PET scans, the data are precorrected for AC events by realtime subtraction of the delayed-window coincidences [7]. The system detects coincidence events during two time windows: "prompt" window and "delayed" window. For each coincidence event in the prompt window, the corresponding sinogram bin is incremented. The statistics of these increments should be well approximated by a Poisson process. However, for coincidence events within the second delayed window, the corresponding sinogram bin is decremented, so the 
resultant "precorrected" measurements are not Poisson. Since prompt events and delayed events are independent Poisson processes, the precorrected measurements correspond to the difference of two independent Poisson random variables with variance equal to the sum of the means of the two random variables. In other words, randoms subtraction compensates in mean for $\mathrm{AC}$ events, but it also increases the variance of the measurement by an amount equal to the mean of AC events.

Let $\underline{Y}=\left[Y_{1}, \ldots, Y_{N}\right]^{\prime}$ denote the vector of precorrected measurements. The precorrected measurement for the $n$th coincidence detector pair is:

$$
Y_{n}=Y_{n}^{\text {prompt }}-Y_{n}^{\text {delay }},
$$

where $Y_{n}^{\text {prompt }}$ and $Y_{n}^{\text {delay }}$ are the number of coincidences within the prompt and delayed windows, respectively. Let $\mu=\left[\mu_{1}, \ldots, \mu_{M}\right]^{\prime}$ denote the vector of unknown linear attenuation coefficients. For transmission scans, we assume that $Y_{n}^{\text {prompt }}$ and $Y_{n}^{\text {delay }}$ are statistically independent Poisson random variables with means $\bar{y}_{n}^{p}$ and $\bar{y}_{n}^{\mathrm{d}}$ respectively as:

$$
\begin{aligned}
\mathrm{E}\left\{Y_{n}^{\text {prompt }}\right\} & =\bar{y}_{n}^{\mathrm{p}}(\mu)=b_{n} e^{-l_{n}(\mu)}+r_{n} \\
\mathrm{E}\left\{Y_{n}^{\text {delay }}\right\} & =\bar{y}_{n}^{\mathrm{d}}=r_{n},
\end{aligned}
$$

where $l_{n}(\mu)=\sum_{j=1}^{M} a_{n j} \mu_{j}$ is the total attenuation between $n$th detector pair. The $a_{n j} \geq 0$ factors have units of length and describe the tomographic system geometry. The $b_{n}>0$ factors denote the blank scan counts and the $r_{n} \geq 0$ factors denote the mean of $\mathrm{AC}$ events.

Since $Y_{n}^{\text {prompt }}$ and $Y_{n}^{\text {delay }}$ are statistically independent and Poisson:

$$
\begin{aligned}
\mathrm{E}\left\{Y_{n}\right\} & =\bar{y}_{n}^{\mathrm{p}}(\mu)-\bar{y}_{n}^{\mathrm{d}}=b_{n} e^{-l_{n}(\mu)}, \\
\operatorname{Var}\left\{Y_{n}\right\} & =\bar{y}_{n}^{\mathrm{p}}(\mu)+\bar{y}_{n}^{\mathrm{d}}=b_{n} e^{-l_{n}(\mu)}+2 r_{n} .
\end{aligned}
$$

\section{Exact Log-Likelihood}

Let $\underline{y}=\left[y_{1}, \ldots, y_{N}\right]^{\prime}$ be a realization of statistically independent random variables $\underline{Y}$ given in (1). Under the usual assumption of independence between different rays, one can express the exact distribution of $\underline{Y}$ using total probability:

$$
\begin{aligned}
P(\underline{Y}=\underline{y} ; \mu) & =\prod_{n=1}^{N} \sum_{m=0}^{\infty} P\left(Y_{n}^{\text {prompt }}=y_{n}+m ; \mu\right) P\left(Y_{n}^{\text {delay }}=m\right) \\
& =\prod_{n=1}^{N} \sum_{m=\left\lfloor-y_{n}\right\rfloor_{+}}^{\infty} \frac{\left[\bar{y}_{n}^{\mathrm{p}}(\mu)\right]^{y_{n}+m} e^{-\bar{y}_{n}^{\mathrm{p}}(\mu)}}{\left(y_{n}+m\right) !} \frac{r_{n}^{m} e^{-r_{n}}}{m !},
\end{aligned}
$$

where $\lfloor x\rfloor_{+}=x$ if $x>0$ and is 0 otherwise. The exact log-likelihood for $\mu$ becomes

$$
\begin{aligned}
L(\mu) & =\log P(\underline{Y}=\underline{y} ; \mu) \\
& =\sum_{n=1}^{N} \log \left(\sum_{m=\left[-y_{n}\right\rfloor_{+}}^{\infty} \frac{\left[\bar{y}_{n}^{\mathrm{p}}(\mu)\right]^{y_{n}+m}}{\left(y_{n}+m\right) !} \frac{r_{n}^{m}}{m !}\right)-\left(\bar{y}_{n}^{\mathrm{p}}(\mu)+r_{n}\right) .
\end{aligned}
$$


Since image reconstruction is ill conditioned, usually one includes a roughness penalty $R(\mu)$ in the objective function. From the Bayesian point of view, this roughness penalty can be thought as a log-prior for $\mu$. Combining this penalty with the log-likelihood yields a penalized-likelihood objective function:

$$
\Phi(\mu)=L(\mu)-R(\mu) .
$$

The goal is to estimate $\mu$ by maximizing $\Phi(\mu)$ over the nonnegative cone:

$$
\hat{\mu}=\arg \max _{\mu \geq 0} \Phi(\mu) .
$$

Since the exact log-likelihood function (5) contains infinite summations, the above maximization is intractable. The following two sections develop tractable yet accurate approximations to $L(\mu)$.

\section{Simple Approximations to the Exact Log-Likelihood}

In this section, we first review the conventional approximations to $L(\mu)$ : the WLS model and the conventional OP model. Then we introduce the SP model [14].

\subsection{Quadratic Approximations}

The quadratic approximation to the exact log-likelihood function results in the data-weighted least squares objective function $L_{W L S}(\mu)$ [12]:

$$
L_{W L S}(\mu)=-\frac{1}{2} \sum_{n=1, y_{n}>0}^{N}\left(l_{n}(\mu)-\hat{l}_{n}\right)^{2} \frac{1}{\hat{\sigma}_{n}^{2}},
$$

where $\hat{l}_{n}=\log \left(\frac{b_{n}}{y_{n}}\right)$ is the method-of-moments estimate of the line integral of the attenuation $l_{n}(\mu)$ and $\hat{\sigma}_{n}^{2}=\frac{y_{n}+2 r_{n}}{y_{n}^{2}}$. The $n$th weighting factor $\hat{\sigma}_{n}^{2}$ is an estimate of the variance of $\hat{l}_{n}\left(y_{n}\right)$ based on a second-order Taylor expansion around $\hat{l}_{n}\left(\bar{y}_{n}\right)$. This weighting is critical for the WLS method. The errors corresponding to projections with large values of $y_{n}$ are weighted more heavily. These projections pass through less dense objects and consequently have higher SNR values.

Alternatively, the choice of $\hat{\sigma}_{n}^{2}=1$ results in the unweighted least-squares (ULS) approach, which leads to much higher variance.

\subsection{Ordinary Poisson (OP) Approximation}

The conventional approach is to assume (approximate) that $\left\{Y_{n}\right\}_{n=1}^{N}$ are distributed as independent Poisson random variables with mean $\bar{y}_{n}=b_{n} e^{-l_{n}(\mu)}$, i.e.:

$$
P(\underline{Y}=\underline{y} ; \mu) \approx \prod_{n=1}^{N} \frac{\left[\bar{y}_{n}(\mu)\right]^{y_{n}} e^{-\bar{y}_{n}(\mu)}}{y_{n} !} .
$$


The log-likelihood corresponding to this OP approximation is:

$$
\begin{aligned}
L_{O P}(\mu) & =\sum_{n=1}^{N} y_{n} \log \bar{y}_{n}(\mu)-\bar{y}_{n}(\mu) \\
& =\sum_{n=1}^{N} y_{n} \log \left(b_{n} e^{-l_{n}(\mu)}\right)-b_{n} e^{-l_{n}(\mu)}
\end{aligned}
$$

disregarding the constants independent of $\mu$.

\subsection{Shifted Poisson (SP) Approximation}

A better approach is to match both the first and second order moments by approximating the quantities $\left\{Y_{n}+2 r_{n}\right\}_{n=1}^{N}$ as having Poisson distributions with means $\left\{\bar{y}_{n}(\mu)+2 r_{n}\right\}$. This model leads to our proposed SP objective function:

$$
\begin{aligned}
L_{S P}(\mu) & =\sum_{n=1}^{N}\left(y_{n}+2 r_{n}\right) \log \left(\bar{y}_{n}(\mu)+2 r_{n}\right)-\left(\bar{y}_{n}(\mu)+2 r_{n}\right), \\
& =\sum_{n=1}^{N}\left(y_{n}+2 r_{n}\right) \log \left(b_{n} e^{-l_{n}(\mu)}+2 r_{n}\right)-\left(b_{n} e^{-l_{n}(\mu)}+2 r_{n}\right) .
\end{aligned}
$$

Note that although both $L_{W L S}$ and $L_{S P}$ match two moments, in WLS the second moment of $\hat{l}_{n}\left(y_{n}\right)$ is "fixed" independently of $\mu$, whereas in the SP model the moments vary with $\bar{y}_{n}(\mu)$ appropriately.

We have previously shown empirically that this model better agrees with the exact log-likelihood than either the WLS or OP model [14]. Next we provide an analytical result that corroborates those results.

\subsection{Variance Analysis}

To analyze the variance of each estimator, we applly the analytic approximations suggested in [2]. If $\bar{Y}=\mathrm{E}\{\underline{Y}\}$, then using a first order Taylor expansion of $\hat{\mu}(\underline{Y})$ results in the following approximation to the covariance of $\hat{\mu}[2]$ :

$$
\operatorname{Cov}\{\hat{\mu}\} \approx P \operatorname{Cov}\{\underline{Y}\} P^{T}
$$

where $P=\left[-\nabla^{20} \Phi(\breve{\mu}, \bar{Y})\right]^{-1} \nabla^{11} \Phi(\breve{\mu}, \bar{Y})$ and $\breve{\mu}=\arg \max _{\mu} \Phi(\mu, \bar{Y})$.

We apply (11) to find approximate expressions for the variance of the maximum likelihood estimators: $\hat{\mu}_{O P}=\arg \max _{\mu} L_{O P}(\mu)$ and $\hat{\mu}_{S P}=\arg \max _{\mu} L_{S P}(\mu)$. For this purpose we considered a highly simplified version of transmission tomography where the unknown is a scalar parameter, i.e. $p=1$. This simplified problem provides insight into the estimator bias and variance without the undue 
notation of the multi-parameter case. The objective functions used here can be expressed in the form:

$$
\Phi(\mu, Y)=\sum_{n=1}^{N} h_{n}(\mu, Y)
$$

Since the measurements are statistically independent, for the scalar problem the above approximation (11) reduces to:

$$
\operatorname{Var}\{\hat{\mu}\} \approx\left(\sum_{n=1}^{N} \frac{\partial^{2} h_{n}(\check{\mu}, \bar{Y})}{\partial \mu^{2}}\right)^{-2} \sum_{n=1}^{N}\left[\frac{\partial^{2} h_{n}(\check{\mu}, \bar{Y})}{\partial \mu \partial Y_{n}}\right]^{2} \operatorname{Var}\left\{Y_{n}\right\}
$$

With some tedious algebra, one can derive the following approximate expressions for variance of $\hat{\mu}_{O P}$ and $\hat{\mu}_{S P}$ :

$$
\begin{aligned}
& \operatorname{Var}\left\{\hat{\mu}_{O P}\right\} \approx \frac{\sum_{n=1}^{N} a_{n}^{2}\left(\bar{y}_{n}\left(\mu_{t}\right)+2 r_{n}\right)}{\left(\sum_{n=1}^{N} a_{n}^{2} \bar{y}_{n}\left(\mu_{t}\right)\right)^{2}} \\
& \operatorname{Var}\left\{\hat{\mu}_{S P}\right\} \approx\left[\sum_{n=1}^{N} \frac{a_{n}^{2} \bar{y}_{n}\left(\mu_{t}\right)^{2}}{\bar{y}_{n}\left(\mu_{t}\right)+2 r_{n}}\right]^{-1},
\end{aligned}
$$

where $\mu_{t}$ denotes the true attenuation coefficient value and $\bar{y}_{n}(\mu)=b_{n} e^{-a_{n} \mu}$.

Letting $s_{n}=a_{n}^{2} \bar{y}_{n}\left(\mu_{t}\right)$ and $t_{n}=a_{n}^{2}\left(\bar{y}_{n}\left(\mu_{t}\right)+2 r_{n}\right)$, one can rewrite $(13)$ and (14) as:

$$
\frac{1}{\operatorname{Var}\left\{\hat{\mu}_{O P}\right\}} \approx \frac{\left(\sum_{n} s_{n}\right)^{2}}{\sum_{n} t_{n}}, \quad \frac{1}{\operatorname{Var}\left\{\hat{\mu}_{S P}\right\}} \approx \sum_{n} \frac{s_{n}^{2}}{t_{n}}
$$

Let $\underline{a}, \underline{b} \in \mathbb{R}^{n}$ such that $a_{n}=\frac{s_{n}}{\sqrt{t_{n}}}, b_{n}=\sqrt{t_{n_{b}}}$. Using Cauchy-Schwarz inequality: $\left|\underline{a}^{T} \underline{b}\right| \leq\|\underline{a}\|_{2}\|\underline{b}\|_{2}$,

$$
\begin{aligned}
\sum_{n} s_{n} & \leq\left(\sum_{n} \frac{s_{n}^{2}}{t_{n}}\right)^{\frac{1}{2}}\left(\sum_{n} t_{n}\right)^{\frac{1}{2}} \\
& \left(\sum_{n} \frac{s_{n}^{2}}{t_{n}}\right)^{-1} \leq \frac{\sum_{n} t_{n}}{\left(\sum_{n} s_{n}\right)^{2}}
\end{aligned}
$$

so that within the accuracy of (11):

$$
\operatorname{Var}\left\{\hat{\mu}_{S P}\right\} \leq \operatorname{Var}\left\{\hat{\mu}_{O P}\right\}
$$

with equality if and only if $r_{n} / \vec{y}_{n}$ ratios are equal. For PET systems, these ratio terms are never constant, and in fact can be quite disparate. Thus we have shown the following result: the variance of the SP estimator will always be lower than the variance of the OP estimator. 


\section{Saddle-point (SD) Approximation}

An alternative to the previous approximations for the exact pmf (4) of precorrected measurements is to make second order Taylor series approximations in the $z$-transform domain (i.e. on the probability generating function) and then to carry out the inverse transform. For this purpose, we have adopted the saddlepoint method $[5,13]$.

Let $U \sim \operatorname{Poisson}(\alpha), V \sim \operatorname{Poisson}(\beta)$ and $Y=U-V$ with pmf's $P_{U}(k), P_{V}(k)$ and $P_{Y}(k)$ respectively. The generating function of $Y$ is:

$$
G_{Y}(z)=\sum_{k} z^{k} P_{Y}(k)=G_{U}(z) G_{V}\left(z^{-1}\right)
$$

where $G_{U}(z)=\exp (\alpha(z-1))$ and $G_{V}(z)=\exp (\beta(z-1))$. In terms of the generating function, $P_{Y}(k)$ is given by the contour integral

$$
P_{Y}(k)=\frac{1}{2 \pi j} \oint_{C^{+}} z^{-k-1} G_{Y}(z) d z=\frac{1}{2 \pi j} \oint_{C^{+}} e^{\Phi_{k}(z)} d z,
$$

where $j=\sqrt{-1}$ and the contour $C^{+}$must lie in the region of convergence of $G_{Y}(z)$ and enclose the origin, and

$$
\begin{aligned}
\Phi_{k}(z) & =-(k+1) \log (z)+\alpha(z-1)+\beta\left(z^{-1}-1\right) \\
\frac{d \Phi_{k}(z)}{d z} & =\Phi_{k}^{(1)}(z)=-\frac{(k+1)}{z}+\alpha-\frac{\beta}{z^{2}} \\
\frac{d^{2} \Phi_{k}(z)}{d z^{2}} & =\Phi_{k}^{(2)}(z)=\frac{(k+1)}{z^{2}}+\frac{2 \beta}{z^{3}} .
\end{aligned}
$$

We observe that $\Phi_{k}(z)$ (and hence the integrand $e^{\Phi_{k}(z)}$ ) is convex for $z \in \mathbb{R}$, $z>0$ and $k \geq 0$. The integrand has a minimum at $x_{o} \in \mathbb{R}, x_{o}>0$ which is called the saddle point, i.e.:

$$
\Phi_{k}^{(1)}\left(x_{o}\right)=-\frac{(k+1)}{x_{o}}+\alpha-\frac{\beta}{x_{o}^{2}}=0 \text { and } x_{o}>0
$$

which yields

$$
x_{o}=\frac{(k+1)+v_{k}}{2 \alpha}=\frac{2 \beta}{-(k+1)+v_{k}},
$$

where $v_{k}=x_{o}^{2} \Phi_{k}^{(2)}\left(x_{o}\right)=\sqrt{(|k|+1)^{2}+4 \alpha \beta}$.

Following [5], we deform the contour $C^{+}$in (16) into a vertical line through saddle point $x_{0}$, as $z=x_{0}+j y,-\infty<y<\infty$ and a semicircle around the left half plane at infinity. This contour is permissible for $k \geq 0$, since the only singularities of the integrand are at $z=0$ and $z=\infty+j 0$. If $|z| \rightarrow \infty$ for $\Re[z]<$ $x_{0}$ then $e^{\Phi_{k}(z)} \rightarrow 0$. Hence the contribution of the semicircle around the left half plane at infinity vanishes and (16) reduces to

$$
P_{Y}(k)=\frac{1}{2 \pi} \int_{-\infty}^{\infty} e^{\Phi_{k}\left(x_{o}+j y\right)} d y
$$


Expanding $\Phi_{k}(z)$ in Taylor's series around $z=x_{o}$, one obtains:

$$
\begin{aligned}
\exp \left[\Phi_{k}(z)\right] & =\exp \left[\Phi_{k}\left(x_{o}\right)+\frac{1}{2} \Phi_{k}^{(2)}\left(x_{o}\right)\left(z-x_{o}\right)^{2}+\sum_{l=3}^{\infty} \frac{1}{l !} \Phi_{k}^{(l)}\left(x_{o}\right)\left(z-x_{o}\right)^{l}\right] \\
& =\exp \left[\Phi_{k}\left(x_{o}\right)+\frac{1}{2} \Phi_{k}^{(2)}\left(x_{o}\right)\left(z-x_{o}\right)^{2}\right]\left[1+\frac{\Phi_{k}^{(3)}\left(x_{o}\right)}{6}\left(z-x_{o}\right)^{3}+\ldots\right]
\end{aligned}
$$

since $\Phi_{k}^{(1)}\left(x_{o}\right)=0$. The integral (18) becomes

$$
\begin{aligned}
P_{Y}(k) & =\frac{e^{\Phi_{k}\left(x_{o}\right)}}{2 \pi} \int_{-\infty}^{\infty} e^{\frac{1}{2} \Phi_{k}^{(2)}\left(x_{o}\right)(j y)^{2}}\left[1+\frac{\Phi_{k}^{(3)}\left(x_{o}\right)}{6}(j y)^{3}+\ldots\right] d y \\
& =\frac{e^{\Phi_{k}\left(x_{o}\right)}}{\sqrt{2 \pi \Phi_{k}^{(2)}\left(x_{o}\right)}}[1+R]=\frac{x_{o}^{-k} e^{v_{k}-\alpha-\beta}}{\sqrt{2 \pi v_{k}}}[1+R]
\end{aligned}
$$

where

$$
R=\frac{\Phi_{k}^{(4)}\left(x_{0}\right)}{8\left[\Phi_{k}^{(2)}\left(x_{0}\right)\right]^{2}}+\ldots
$$

Using the algorithm by Rice [11], the residuum $\mathrm{R}$ can be written as:

$$
R=\frac{1}{24(k+1)}\left[\frac{-5+12 \sqrt{1+\eta}-9(1+\eta)}{(1+\eta)^{3 / 2}}\right]+O\left[\left(\frac{1}{k+1}\right)^{2}\right]
$$

where $\eta=\frac{4 \alpha \beta}{(k+1)^{2}}$. The residuum asymptotically goes to zero as $k \rightarrow \infty$ and more importantly we have observed empirically that the approximation error is negligibly small even for very small values of $k$. Neglecting $\mathbf{R}$ in (19) results in our saddle-point approximation for the $\operatorname{pmf} P_{Y}(k)$ as:

$$
P_{Y}(k) \simeq P_{Y}^{s}(k)=\frac{x_{o}^{-k} e^{v_{k}-\alpha-\beta}}{\sqrt{2 \pi v_{k}}}, k \geq 0 .
$$

For $k<0$ the integrand in (16) is not guaranteed to be convex for $z>0$. Moreover, the integrand does not vanish along the semicircle around the left half plane at infinity. Thus we use the change of variables $w=1 / z$ in (16), so that:

$$
P_{Y}(k)=\frac{1}{2 \pi j} \oint_{C^{+}} w^{k-1} G_{Y}\left(w^{-1}\right) d w=\frac{1}{2 \pi j} \oint_{C^{+}} e^{\check{\Phi}_{k}(w)} d w
$$

where

$$
\check{\Phi}_{k}(w)=(k-1) \log (w)+\alpha\left(w^{-1}-1\right)+\beta(w-1) .
$$

Following similar steps as the case for $k \geq 0$, the saddle point approximation for $k<0$ can be shown to be :

$$
P_{Y}(k) \simeq P_{Y}^{s}(k)=\frac{w_{0}^{k} e^{v_{k}-\alpha-\beta}}{\sqrt{2 \pi v_{k}}}, k<0
$$


where

$$
w_{o}=\frac{-(k-1)+v_{k}}{2 \beta}=\frac{2 \alpha}{(k-1)+v_{k}} .
$$

Thus, combining (20) and (22) the saddle-point (SD) approximation for the log-likelihood (5) is:

$$
\begin{aligned}
L_{S D}(\mu) & =\sum_{n=1}^{N} \log P_{Y}^{s}\left(y_{n} ; \bar{y}_{n}(\mu)\right) \\
& =\sum_{n=1}^{N} h_{n}^{s}(\mu)
\end{aligned}
$$

where

$$
h_{n}^{s}(\mu)=\left\{\begin{array}{l}
y_{n} \log \left(\frac{\bar{y}_{n}(\mu)+r_{n}}{y_{n}+1+u_{n}(\mu)}\right)-\bar{y}_{n}(\mu)+u_{n}(\mu)-\frac{1}{2} \log u_{n}(\mu), y_{n} \geq 0 \\
y_{n} \log \left(\frac{\bar{y}_{n}(\mu)+r_{n}}{y_{n}-1+u_{n}(\mu)}\right)-\bar{y}_{n}(\mu)+u_{n}(\mu)-\frac{1}{2} \log u_{n}(\mu), y_{n}<0
\end{array}\right.
$$

with $u_{n}(\mu)=\sqrt{\left(\left|y_{n}\right|+1\right)^{2}+4\left(\bar{y}_{n}(\mu)+r_{n}\right) r_{n}}$ and disregarding constants independent of $\mu$.

Note that this approximation is considerably simpler than the exact loglikelihood (5), since no infinite sums or factorials are needed. Nevertheless, it is remarkably accurate as shown below. Also, one can observe that as $r_{n} \rightarrow 0$, $h_{n}^{s}(\mu) \rightarrow\left[y_{n} \log \bar{y}_{n}(\mu)-\bar{y}_{n}(\mu)\right]=L_{O P}(\mu)$ (to within constants independent of $\mu$ ), which is expected because for $r_{n}=0$ the ordinary Poisson model is appropriate.

Fig. 4 shows a representative comparison of the exact log-likelihood function and the approximations for noiseless data as a function of $\mu$. Although $L_{S P}(\mu)$ fits the exact $\log$-likelihood better than $L_{W L S}(\mu)$ and $L_{O P}(\mu)$, clearly $L_{S D}(\mu)$ has the best agreement with the exact log-likelihood $L(\mu)$. In a large number of additional comparisons not shown due to space considerations, we have observed that $L_{S D}(\mu)$ agrees remarkably well with the exact $\log$-likelihood $L(\mu)$ and clearly better than the other models.

\section{2D Simulations}

To study bias and variance properties of the estimators based on the above approximations, we performed 2D simulations. For $\mu$ we used the synthetic attenuation map shown in Fig. 1, which represents a human abdomen with linear attenuation coefficient $0.0096 / \mathrm{mm}$. The image was a 128 by 64 array of 4.5 mm pixels. We simulated a PET transmission scan with 192 radial bins and 256 angles uniformly spaced over 180 degrees. The $a_{n j}$ factors correspond to 6 $\mathrm{mm}$ wide strip integrals on $3 \mathrm{~mm}$ center-to-center spacing. The $b_{n}$ factors were 
generated using pseudo-random log-normal variates with standard deviation of 0.3 to account for detector efficiency variations, and scaled so that $\sum_{n} \bar{y}_{n}$ was one million counts. The $r_{n}$ factors corresponded to a uniform field of $5 \%$ random coincidences. Pseudo-random transmission measurements were generated according to (2) and (3). For regularization, we used the modified quadratic penalty [4], which matches the spatial resolution of different estimators.

We generated 100 independent realizations of the transmission measurements. For each measurement realization, an estimate of the attenuation map was reconstructed using 20 iterations of the grouped-coordinate ascent algorithms [3] applied to the objective functions (8), (10), (11) and (23). We computed both the sample mean and sample standard deviation images for all methods.

Fig. 2 shows horizontal profiles through the sample mean images. These profiles show that WLS is systematically negatively biased, whereas the OP, SP and $\mathrm{SD}$ models are free of systematic bias. (The overshoot at the edges is due to the quadratic penalty used in the reconstruction. Even with noiseless data, this blurring effect will still be present.)

To study the variance, we computed the ratio of sample standard deviation images of different estimators, over all interior pixels. Fig. 3 shows the histogram of the standard deviation ratios. The OP model yields, on the average, $20 \%$ higher standard deviation than the both SP and SD models. In other words, to achieve the same noise level, the OP method would require about $40 \%$ greater scan time.

Although the standard deviation values could be decreased by using higher count rates, the ratio of standard deviations of different estimators will remain approximately same for higher count rates [2].

We performed additional simulations using the thorax phantom with nonuniform attenuation [14]. The results were comparable.

\section{Estimates of the $\mathrm{AC}$ rates $\left(\hat{r}_{n}\right)$}

One needs to know the mean of the AC events $\left(r_{n}\right)$ in order to compute $\mathrm{L}_{S P}(\mu)$ and $L_{S D}(\mu)$. Since the $r_{n}$ terms are not readily available from the real (precorrected) data, some estimates of the randoms must be used.

Fig. 5 displays the scatter plot of real delayed coincidence sinograms for blank scan and transmission scan data. Each point in the plot corresponds to a specific detector pair. The similarity of both delayed coincidence measurements suggests that one can acquire the delayed coincidence events during the blank scan and use them (after properly normalizing for different scan durations) as an estimate of the $\mathrm{AC}$ rates for transmission scans performed on the same PET system. We performed additional simulations (not shown) in which we substituted a simple constant for $r_{n}$ rather than the true values into the SP and SD objective functions. This approximation resulted in only a slight increase in the standard deviation (around 2\%) of the SP and SD estimates without any systematic bias. These results demonstrate that both the SP and SD approximations are robust to errors in the $r_{n}$ estimates. 


\section{Discussion}

AC events are a primary source of background noise in positron emission tomography. After the $\mathrm{AC}$ events are precorrected, the measurement statistics are no longer Poisson. For transmission scans, WLS method and ML method based on ordinary Poisson (OP) model lead to systematic bias and higher variance, respectively, compared to our proposed shifted Poisson (SP) model for measurement statistics which matches both the first and second-order moments.

We proposed a new approximation for the exact log-likelihood which is derived using saddle-point approximation to the pmf of precorrected measurements. Both the analysis of the error term and the log-likelihood plots and 1D simulations (not shown due to space considerations) show that the new approximation agrees very closely with the exact log-likelihood compared to previous approximations.

2D simulations show that both SP and SD models perform very closely. They are both free of systematic bias and yield reduced standard deviation (about $20 \%$ ) compared to OP model. As we observed very close agreement between exact $\log$-likelihood and SD approximation both from the $\log$-likelihood plots and 1D simulations, we were expecting SD method to perform better than SP method. However, for the 2D simulations reported here, the SP method performed as well as SD method. Thus the SP method is particularly attractive since it requires comparable computation to OP method but has reduced variance. We plan to compare the SD and SP methods to the uniform Cramer-Rao bounds [6].

The high correlation between delayed coincidence events of blank and transmission scans suggest that one can use AC rates estimated from blank scans. We have seen that even using constant $\mathrm{AC}$ rates in $2 \mathrm{D}$ simulations resulted in only a slight increase in the standard deviation without any systematic bias. Thus the proposed SP and SD methods are robust enough for practical use.

We plan to apply the proposed method to emission tomography, where even higher $\mathrm{AC}$ rates than the transmission tomography are common, particularly in 3D PET. Moreover, in 3D PET, very large data sets are likely to preclude separate acquisition of random coincidences, so the real-time subtraction methods are usually used for emission scans. So the potential benefit of the proposed models should be even greater.

\section{References}

1. M E Casey and E J Hoffman. Quantitation in positron emission computed tomography: $7 \mathrm{~A}$ technique to rednce noise in accidental coincidence measurements and coincidence efficiency calibration. J. Comp. Assisted Tomo., 10(5):845-850, 1986.

2. J A Fessler. Mean and variance of implicitly defined biased estimators (such as penalized maximum likelihood): Applications to tomography. IEEE Tr. Im. Proc., 5(3):493-506, March 1996.

3. J A Fessler, E P Ficaro, N H Clinthorne, and K Lange. Grouped-coordinate ascent algorithms for penalized-likelihood transmission image reconstruction. IEEE $\mathrm{Tr}$. Med. Im., 16, April 1997. To appear. 
4. J A Fessler and W L Rogers. Spatial resolution properties of penalized-likelihood image reconstruction methods: Space-invariant tomographs. IEEE Tr. Im. Proc., 5(9):1346-58, September 1996.

5. C Helstrom. Approximate evaluation of detection probabilities in radar and optical communications. IEEE Tr. Aero. Elec. Sys., 14(4):630-40, 1978.

6. A O Hero, J A Fessler, and M Usman. Exploring estimator bias-variance tradeoffs using the uniform CR bound. IEEE Tr. Sig. Proc., 44(8):2026-41, August 1996.

7. E J Hoffman, S C Huang, M E Phelps, and D E Kuhl. Quantitation in positron emission computed tomography: 4 Effect of accidental coincidences. J. Comp. Assisted Tomo., 5(3):391-400, 1981.

8. E Ü Mumcuoğlu, R M Leahy, and S R Cherry. Bayesian reconstruction of PET images: methodology and performance analysis. Phys. Med. Biol., 41:1777-1807, 1996.

9. J M Ollinger and J A Fessler. Positron emission tomography. IEEE Signal Proc. Mag., 14(1):43-55, January 1997.

10. D G Politte and D L Snyder. Corrections for accidental coincidences and attenuation in maximum-likelihood image reconstruction for positron-emission tomography. IEEE Tr. Med. Im., 10(1):82-89, March 1991.

11. S O Rice. Uniform asymptotic expansions for saddle point integrals-application to a probability distribution occuring in noise theory. Bell Syst. Tech J., 47:1971-2013, November 1968.

12. K Sauer and $\mathrm{C}$ Bouman. A local update strategy for iterative reconstruction from projections. IEEE Tr. Sig. Proc., 41(2):534-548, February 1993.

13. D L Snyder, C W Helstrom, A D Lanterman, M Faisal, and R L White. Compensation for readout noise in CCD images. J. Opt. Soc. Amer. Ser. A, 12(2):272-83, February 1995.

14. M Yavuz and J A Fessler. Objective functions for tomographic reconstruction from randoms-precorrected PET scans. In Proc. IEEE Nuc. Sci. Symp. Med. Im. Conf., 1996. To appear.

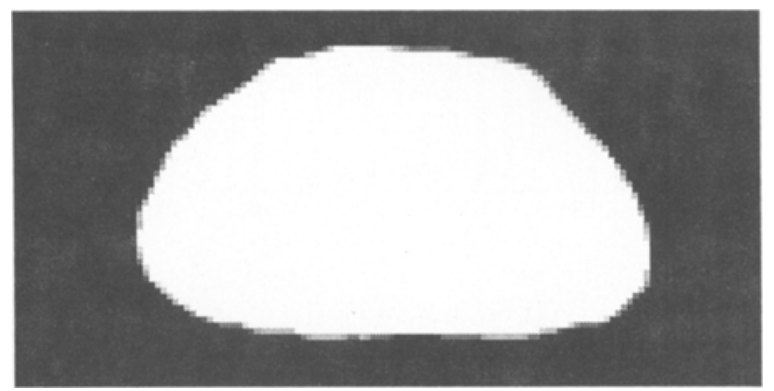

Fig. 1. Simulated abdomen attenuation map. 


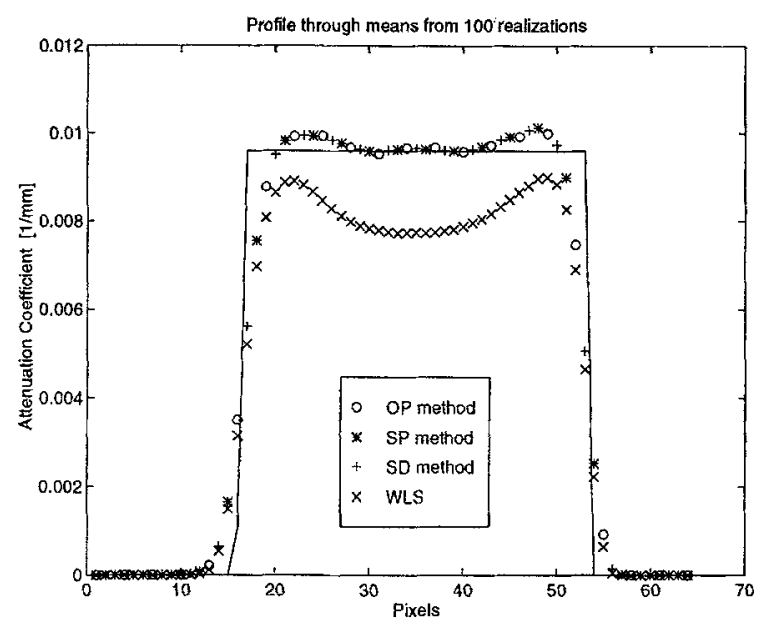

Fig. 2. Horizontal profile through the sample mean images for abdomen phantorn. The WLS method has a systematic negative bias. The ordinary Poisson (OP), shifted Poisson (SP) and saddle-point (SD) methods are free of this systematic negative bias.
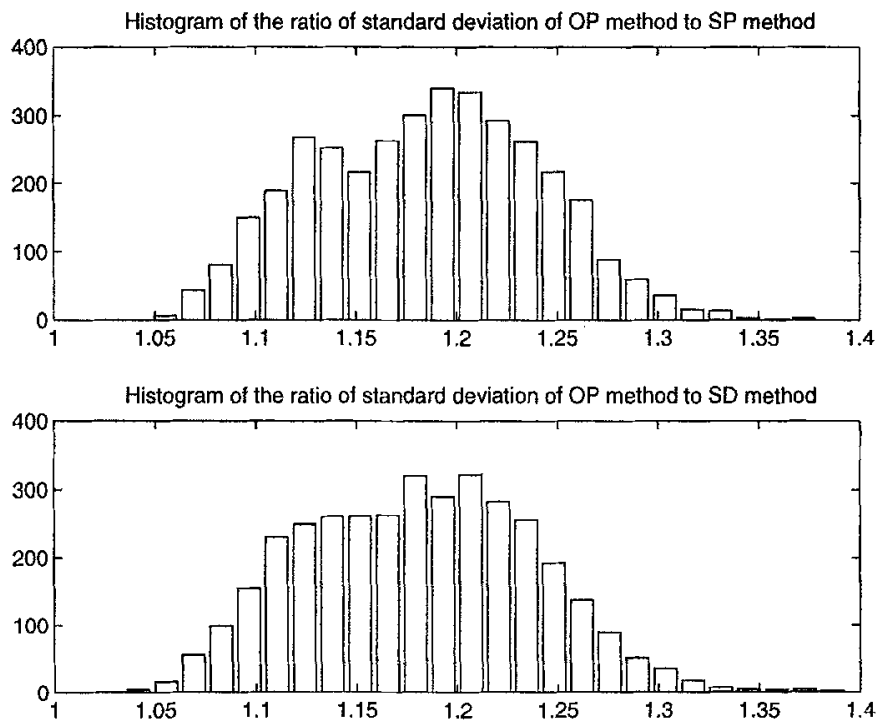

Fig. 3. Histogram of the ratio of standard deviations in reconstructions of the abdomen phantom. The ordinary Poisson (OP) method yields, on the average, 20\% higher standard deviation than the proposed shifted Poisson (SP) and saddle-point (SD) methods. 


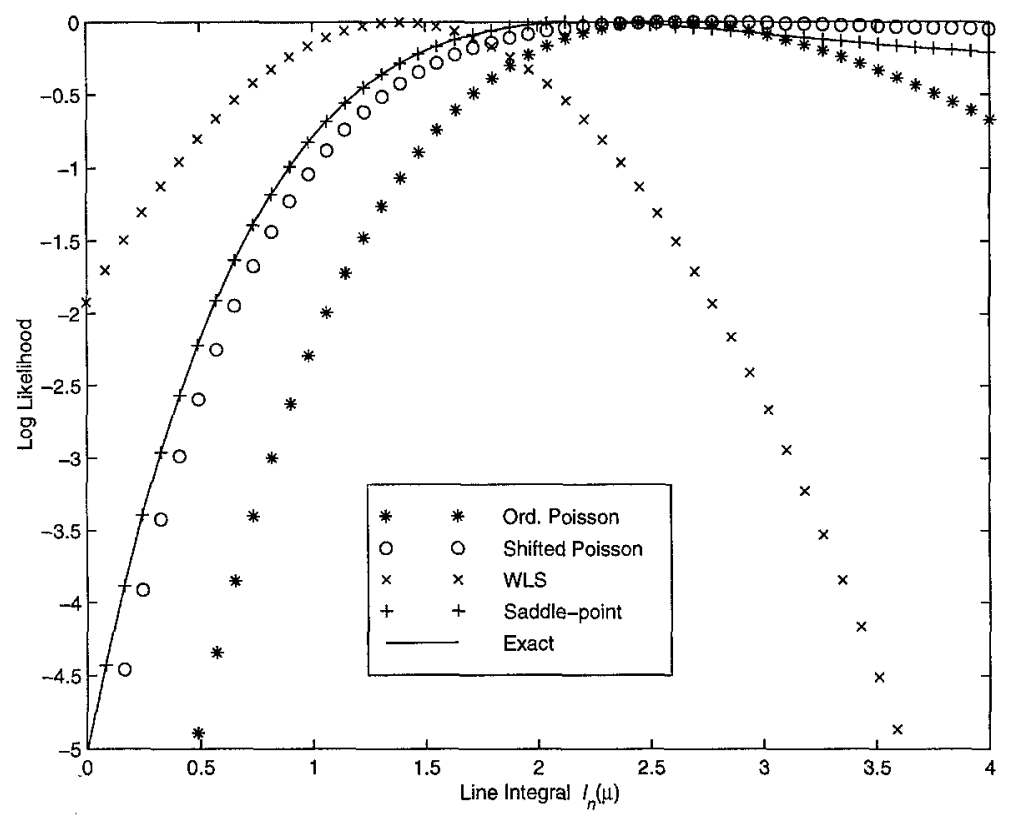

Fig. 4. Representative comparison of exact log-likelihood function with objective functions of different models as a function of line integral $l_{n}(\mu)$. Randoms rate is $5 \%$. The proposed saddle-point approximation agrees with exact log-likelihood significantly better than the other models.

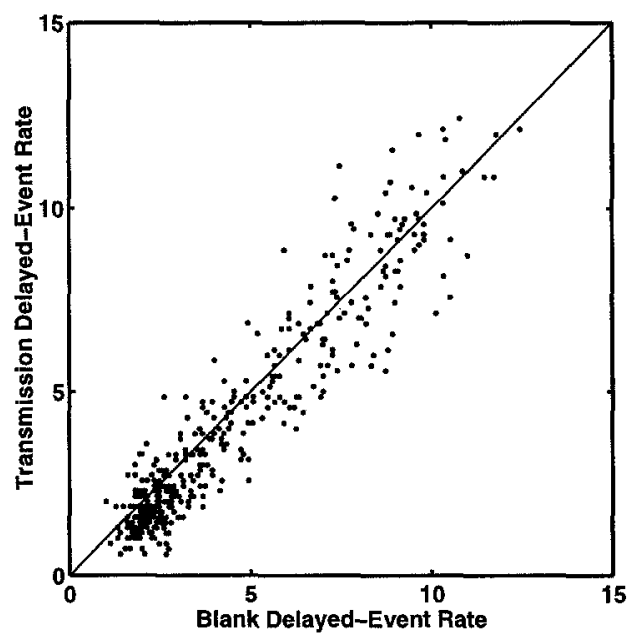

Fig. 5. Scatter plot of delayed coincidence event of blank and transmission scans. 